\title{
Ia convergenza in media del quarto ordine dei polinomi di interpolazione di Lagrange relativi ad un particolare sistema di punti interpolanti.
}

\author{
Nota di Luigi Merli (a Firenze) (*).
}

Sunto. - Sia $\left\{\mathrm{I}_{\mathrm{n}}(\mathrm{x}) \mid\right.$ la successione dei polimomi di Lagrange che interpolano $\mathrm{f}(\mathrm{x}),-1 \leq \mathrm{x} \leq 1$, negli zeni del polinonio $d i$ J J peso $\mathrm{p}(\mathrm{x})=\sqrt{\frac{1-\mathrm{x}}{1+\mathrm{x}}} \cdot$ I A. dimostra che se $\mathrm{f}(\mathrm{x})$ soddisfa ad una condizione di LuPSCHIT ai ordine $\alpha>\frac{1}{4}$, si ha:

$$
\lim _{n \rightarrow \infty} \int_{-1}^{1} \sqrt{\frac{1-x}{1+x}}\left(f(x)-L_{n}(x)\right)^{4} d x=0
$$

1. Consideriamo il polinomio di Lagrange

$$
L_{n}(x)=\sum_{k=1}^{n} f\left(x_{k}\right) l_{k}^{(n)}(x)
$$

dove

$$
\begin{array}{cr}
l_{k}^{(n)}(x)=\frac{\omega_{n}(x)}{\omega_{n}{ }^{\prime}\left(x_{k}\right)\left(x-x_{k}\right)}, & (k=1,2, \ldots, n), \\
\omega_{n}(x)=C \prod_{s=1}^{n}\left(x-x_{s}\right) & (c \neq 0),
\end{array}
$$

chr, come è noto, dà il polinomio interpolante di grado non superiore ad $n-1$, che negli $n$ punti $x_{1}, x_{2}, \ldots, x_{n}$, prende rispettivamente i valori

$$
f\left(x_{1}\right), f\left(x_{2}\right), \ldots, f\left(x_{n}\right)\left({ }^{1}\right) .
$$

Alcuni Autori, come. G. Szegö $\left({ }^{2}\right)$, P. ERdös e P. Turà $\left({ }^{3}\right)$, hanno provato che se la $(1)$ è costruita sugli zeri dei polinomi ortogonali $\left\{\omega_{n}(x)\right\}$ relativi

(*) Lavoro eseguito nell' Istitnto Matematico dell' Università di Firenze.

(1) Cfr. per es. E. FELIHEIM. Thérie de la concergence des procédés d'interpolation etc., "Mem. Sc. Math. ", 95 (1939), pag. 3.

(*) G. Szerib, Orthogonal Polynomials. "Am. Math. Coll. Publ.», XXIII (1939), pag. 326.

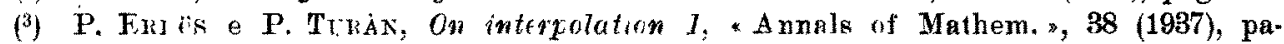
gine 145-155. 
ad un peso $p(x) \geq 0$, ha Inogo la convergenza in media di ordine 2 , relativa a tale peso, per tutte le funzioni $f(x)$ continue; si ha oioe:

$$
\lim _{n \rightarrow \infty} \int_{-1}^{1} p(x)\left[f(x)-L_{n}(x)\right]^{2} d x=0
$$

Recentemente E. Feldherar ( $\left.{ }^{4}\right)$, passando oltre in tale tipo di ricerche, si è occupato della convergenza in media di ordine 4; di studiare cioè quando vale la relazione

$$
\lim _{n \rightarrow \infty} \int_{-1}^{1} p(x)\left[f(x)-L_{n}(x)\right]^{4} d x=0
$$

ed ha provato che, contrariamente a quanto avviene per la convergenza in media di ordine 2, essa non è vera in generale. Egli ha infatti dimostrato che la (2) vale, per tutte le funzioni continue, se i punti di interpolazione sono gli zeri dei polinomi di IACOBI $\omega_{n}(x)=I_{n}\left(-\frac{1}{2},-\frac{1}{2}, x\right)$, ma non in generale per i polinomi $I_{m}(\alpha, \beta, x)$ con $\alpha \neq-\frac{1}{2}, \beta \neq-\frac{1}{2}(\%)$

Noi studieremo in questa Nota il caso del polinomio $\omega_{n}(x)=I_{n}\left(\frac{1}{2},-\frac{1}{2}, x\right)$ e daremo delle condinioni sufficienti cui deve soddjsfare la funzione $f(x)$, oltre alla semplice continuità, perchè valga ancora la (2) e dimostreremo precisa. mente il teorema:

Se $\mathrm{f}(\mathrm{x})$ è una qualunque funzione che soddisfa ad una condizione di Lis. SCHITZ di ordine $\propto>\frac{1}{4}$ ed $\left\{\mathrm{S}_{n}(\mathrm{x})\right\}$ indica la successione dei polinomi di inter.

(4) E. Fondhan, Sur l'orthogonalité des fonctions fondamentales et sur la forte conver. gence en moyenne des polynomes d'interpolation de Lagrange etc., "Bul]. de la Soc. Math. de France ", LXY (1987), pagg. 1-40.

(5) I polinomi di IAcosi $I_{n}(\alpha, \beta, x)$, com's noto, soldisfano l'cquazione differenziale

$\left(1-x^{2}\right) I_{n}{ }^{\prime}(x, \beta, x)+[(\beta-x)-(\beta+x+2) x] I_{n}{ }^{\prime}(x, \beta, x)+n(n+x+\beta+1) I_{n}(x, \beta, x)=0$

e sono ortogonali, per $\alpha>-1, \beta>-1$, rispeffo alla funziono $\eta^{\circ \text { so }} p(x)=(1-x) \propto(1-x) \xi$. Il polinomio $I_{n}\left(-\frac{1}{2},-\frac{1}{2}, x\right)$ è detto polinomio di Tukbrchur di $1^{\text {a }}$ specie ed ha la forma trigonometrica semplicissima $I_{n}\left(-\frac{1}{2},-\frac{1}{2}, x\right)=\cos n \theta, x=\cos \theta$. Sono di $2^{a}$ specie i poli. nomi $I_{n}\left(\frac{1}{2}, \frac{1}{2}, x\right)=\frac{\operatorname{sen} n \theta}{\operatorname{sen} \theta}, x=\cos \theta$. Noi considereremo nella presente Nota il polinomio $I_{n}\left(\frac{1}{2},-\frac{1}{2}, x\right)=\frac{\operatorname{sen}\left(n+\frac{1}{2}\right) \theta}{\operatorname{sen} \frac{\theta}{2}}, x=\cos \theta$ che abbiamo già avuto occasione di studiare in al. cune Note precedenti. [Cfr. per es.: L. MenL, Sulla convergenza in media della formula di interpolaziome di Hermite etc., "Gjorn. 1st. Tt. Attuari", XTI (1941), pagg. 1-6]. 
1. MERII : La coneryman in media del quarto ordine dei polinomi, nee. 280

polazione di LAGRANGE. relativa a tale funzione e costruita sugli zeri dcl polinomio $\mathrm{I}_{\mathrm{n}}\left(\frac{1}{2},-\frac{1}{2}, \mathrm{x}\right)$, si ha:

$$
\lim _{n \rightarrow \infty} \int_{-1}^{1} \sqrt{\frac{1-x}{1+x}}\left[f(x)-L_{n}(x)\right]^{4} d x=0 \quad\left(^{6}\right)
$$

2. Il polinomio $\omega_{n}(x)=I_{n}\left(\frac{1}{2} ;-\frac{1}{2}, x\right)=\frac{\operatorname{sen}\left(n+\frac{1}{2}\right) \theta}{\operatorname{sen} \frac{\theta}{2}}, 0 \leq \theta \leq \pi, x=\cos \theta$, che noi consideriamo, ha gli zeri nei punti dell'intervallo $(-1,1)$

$$
x_{k}=\cos \theta_{k}, \theta_{k}=\frac{2 k \pi}{2 n+1} \quad(k=1,2, \ldots, n)
$$

ed e relativo al peso

Essendo

$$
p(x)=(1-x)^{\frac{1}{2}}(1+x)^{-\frac{1}{2}}
$$

$$
\omega_{n}^{\prime}(x)=l_{n}^{\prime}\left(\frac{1}{2},-\frac{1}{2}, x\right)=-\left(-\frac{1}{2}\right) \cos \left(n+\frac{1}{2}\right) \theta \operatorname{sen} \frac{\theta}{2}-\frac{1}{2} \cos \frac{\theta}{2} \operatorname{sen}\left(n+\frac{1}{2}\right) \theta
$$

e percio

$$
\omega_{n}^{\prime}\left(x_{k}\right)=(-1)^{k+4}\left(n+\frac{1}{2}\right) \frac{1}{\operatorname{sen} \frac{\theta_{k}}{2} \operatorname{sen} \theta_{k}},
$$

si ha, tenuto conto della $\left(1^{\prime}\right)$,

$$
l_{k}^{(n)}(x)=\frac{(-1)^{k+1} \operatorname{sen}\left(n+\frac{1}{2}\right) \theta \operatorname{sen} \frac{\theta_{k}}{2} \operatorname{sen} \theta_{k}}{\left(n+\frac{1}{2}\right) \operatorname{sen} \frac{\theta}{2}\left(\cos \theta-\cos \theta_{k}\right)}, x=\cos \theta .
$$

(6) E noto [efr. G. Szacö, op. cit. in (2), pag. 328] che se la successione $\left|I_{n}(x)\right|$ è co struita sugli zeri di $I_{n}\left(\frac{1}{2},-\frac{1}{2}, x\right)$ ed $f(x)$ ha derirata continua, si ha. uniformemente nell'intervallo $(-1,1)$

$$
\lim _{n \rightarrow \infty} L_{n}(x)=f(x)
$$

In tali condizioni si avrà anche, evidentemente,

$$
\lim _{n \rightarrow \infty} \int_{-1}^{1} p(x)\left[f(x)-L_{n n}(x)\right]^{4} d x=0
$$

sopon di questa Nota i lo studio di tale limite indipendentemente dalla derivabilith di $f(x)$. 
Osserviamo, in primo lnogo, ohe se $P_{n-4}(x)$ indica un polinomio di grado $\leq n-1$, si ha:

$$
\begin{gathered}
\int_{-1}^{1} p(x)\left[f(x)-L_{n}(x)\right]^{4} d x=\int_{-1}^{1} p(x)\left[f(x)-P_{n-1}(x)+P_{n-1}(x)-L_{n}(x)\right]^{4} d x \leq \\
\leq 8 \int_{-1}^{1} p(x)\left[f(x)-P_{n-1}(x)\right]^{4} d x+8 \int_{-1}^{1} f(x)\left[P_{n-1}(x)-L_{n}(x)\right]^{4} d x= \\
=8 \int_{-1}^{1} p(x)\left[f(x)-P_{n-1}(x)\right]^{4} d x+8 \int_{-1}^{1} p(x)\left[\sum_{k=1}^{n} P_{n-1}\left(x_{k}\right) l_{k}^{(n)}(x)-\sum_{k=1}^{n} f\left(x_{k}\right) l_{k}^{(n)}(x)\right]^{4} d x= \\
\left.=8 \int_{-1}^{1} p(x)\left[f(x)-P_{n-1}(x)\right]^{4} d n+\left.8 \int_{-1}^{1} p(x)\right|_{n=1} ^{n}\left[P_{n-1}\left(x_{k}\right)-f\left(x_{k}\right)\right] l_{k}^{(n)}(x)\right]^{4} d x,
\end{gathered}
$$

e posto

(3)

$$
\max _{-1 \leq x \leq 1}\left|f(x)-P_{n-1}(x)\right|=\Delta
$$

si avrà :

(3) $\left.\int_{-1}^{1} p(x)\left[f(x)-L_{n}(x)\right]^{4} d x \leq 8 \pi \Delta^{4}+8 \Delta^{4} \int_{-1}^{1} p(x)\left\{\sum_{k=1}^{n}\left|l_{k}^{(n)}(x)\right|\right\}^{4} d x=8 \Delta^{4} \mid \pi+I(n)\right]$, avendo posto

$$
I(n)=\int_{-1}^{1} p(x)\left\{\sum_{x=1}^{n}\left|l_{k}^{(n)}(x)\right|\right\}^{s} d x
$$

Tenuto conto della $\left(1^{\prime \prime}\right)$ avremo allora, nel nostro caso,

$$
I(n)=2 \int_{i}^{\pi} \frac{1}{\operatorname{sen}^{2} \frac{\theta}{2}}\left|\sum_{k=1}^{n}\right| \frac{\operatorname{sen}\left(n+\frac{1}{2}\right) \theta \operatorname{sen} \frac{\theta_{k}}{2} \operatorname{sen} \theta_{k}}{\left(n+\frac{1}{2}\right)\left(\cos \theta-\cos \theta_{k}\right)}||^{4} d \theta .
$$

Essendo

$$
\operatorname{son} \theta_{h} \leq 2 \operatorname{sen} \frac{\theta+\theta_{k}}{2}
$$

si avrè :

$$
I(n) \leq 2 \int_{0}^{\pi} \frac{1}{\operatorname{sen}^{2} \frac{\theta}{2}}\left|\sum_{k=1}^{n}\right| \begin{aligned}
& \operatorname{sen}\left(n+\frac{1}{2}\right) \theta \operatorname{sen} \frac{\theta_{k}}{2} \\
& \left(n+\frac{1}{2}\right) \operatorname{sen} \frac{\theta-\theta_{k}}{2}
\end{aligned}||^{4} d \theta
$$


L. MERLI: La convergenza in media del quarto ordine dei polinomi, ecc. $\quad 287$

Scindiamo ora l'intervallo di integrazione nei due intervalli parziali $\left(0, \frac{\theta_{1}}{2}\right),\left(\frac{\theta_{1}}{2}, \pi\right)$ e posto

$$
\begin{aligned}
& \left.I_{1}(n)=\int_{\theta}^{\frac{\theta_{1}}{2}} \int_{\operatorname{sen}^{2}{ }_{2}^{\theta}}^{1} \sum_{k=1}^{n} \mid \begin{array}{l}
\operatorname{sen}\left(n+\frac{1}{2}\right) \theta \operatorname{sen} \frac{\theta_{k}}{2} \\
\left(n+\frac{1}{2}\right) \operatorname{sen} \frac{\theta-\theta_{k}}{2}
\end{array}\right\} d \theta \\
& I_{2}(n)=\int_{\theta_{1}}^{\pi} \frac{1}{\operatorname{sen} \frac{\theta}{2}}\left|\sum_{k=1}^{n}\right| \begin{array}{l}
\operatorname{sen}\left(n+\frac{1}{2}\right) \theta \operatorname{sen} \frac{\theta_{k}}{2} \\
\left(n+\frac{1}{2}\right) \operatorname{sen} \frac{\theta-\theta_{k}}{2}
\end{array}||^{4} d \theta,
\end{aligned}
$$

avremo:

(4)

$$
I(n) \leq 2\left[I_{1}(n)+I_{2}(n)\right]
$$

3. Maggiorazione di $\mathrm{I}_{1}(\mathrm{n})$ e di $\mathrm{I}_{2}(\mathrm{n})$.

a) Essendo

$$
\left|\frac{\operatorname{sen}\left(n+\frac{1}{2}\right)}{\operatorname{sen} \frac{\theta}{2}}\right| \leq 2 n+1
$$

tenuto conto che $\dot{e}$, in generale,

avremo:

$$
\left\{\sum_{k=1}^{n}\left|a_{k}\right|\right\}^{4} \leq n^{3} \sum_{k=1}^{n} a_{k}^{4}
$$

$l_{i}(n) \leq \frac{n^{3}}{\left(n+\frac{1}{2}\right)^{4}} \int_{0}^{\frac{\theta_{1}}{2}} \frac{1}{\operatorname{sen}^{2} \frac{\theta}{2}} \sum_{k=1}^{n} \frac{\operatorname{sen}^{4}\left(n+\frac{1}{2}\right) \theta \operatorname{sen}^{4} \frac{\theta_{k}}{2}}{\operatorname{sen}^{4} \cdot \frac{\theta-\theta_{k}}{2}} d \theta \leq \frac{16 n^{3} \pi}{(2 n+1)^{3}} \sum_{k=1}^{n} \frac{\operatorname{sen}^{4} \frac{\theta_{k}}{\operatorname{sen}^{4} \frac{1}{2}\left(\frac{\theta_{i}}{2}-\theta_{k}\right)}}{.}$

Valutiamo ora quest' ultima somma. Essendo $\left(\right.$ per $\left.0<x<\frac{\pi}{2}\right) 1>\frac{\operatorname{sen} x}{x}>\frac{2}{\pi}$, si ha:

$$
\begin{gathered}
\sum_{k=1}^{n} \frac{\operatorname{sen}^{4} \frac{\theta_{k}}{2}}{\operatorname{sen}^{4} \frac{\theta_{1}}{2}-\theta_{k}} \leq \frac{1}{2} \sum_{k=1}^{n} \frac{\theta_{k}{ }^{4}}{\operatorname{sen}^{4} \frac{\frac{\theta_{1}}{2}-\theta_{k}}{2} \cdot\left(\frac{\frac{\theta_{1}}{2}-\theta_{k}}{2}\right)^{4} \cdot\left(\frac{2}{\frac{\theta_{1}}{2}-\theta_{k}}\right)^{4}} \leq \\
\leq \frac{\pi^{4}}{16} \sum_{k=1}^{n} \frac{\theta_{k}{ }^{4}}{\left(\frac{\theta_{1}}{2}-\theta_{k}\right)^{4}}=\frac{\pi^{4}}{16} \sum_{k=1}^{n} \frac{(2 k \pi)^{4}}{(\pi-2 k \pi)^{4}}=\frac{\pi^{4}}{16} \sum_{k=1}^{n} \frac{16 k^{4}}{(2 k-1)^{4}} \leq \pi^{4} \sum_{k=1}^{n} \frac{1}{\left(2-\frac{1}{k}\right)^{4}} \leq \pi^{4} n .
\end{gathered}
$$


Avremo pereio:

(5)

$$
I_{1}(n) \leq i_{1} n
$$

con $C_{1}$ costante assoluta indipendente da $n$.

b) Consideriamo ora

$$
I_{2}(n)=\left.\int_{\frac{\theta_{1}}{2}}^{\pi} \frac{1}{\operatorname{sen}^{2} \frac{H}{2}}\left|\sum_{k=1}^{n} \frac{\operatorname{sen}\left(n+\frac{1}{2}\right) \theta \operatorname{sen} \frac{\theta_{k}}{2}}{\left(n+\frac{1}{2}\right) \operatorname{sen} \frac{\theta-\theta_{k}}{2}}\right|\right|^{*} d \theta .
$$

Essendo

$$
\operatorname{sen}\left(n+\frac{1}{2}\right) \theta=(-1)^{3 n+1} \operatorname{sen}\left(n+\frac{1}{2}\right)\left(\theta-\theta_{k}\right)
$$

avremo:

$$
\left.I_{2}(n) \leq \int_{\frac{\theta_{1}}{2}}^{\pi} 1\left|\operatorname{sen}^{2} \frac{1}{2}\right| \sum_{k=1}^{n}\left|\begin{array}{c}
\operatorname{sen}\left(n+\frac{1}{2}\right)\left(\theta-\theta_{k}\right. \\
\left(n+\frac{1}{2}\right) \operatorname{sen} \theta-\theta_{k}
\end{array}\right|\right\}^{k} d \theta
$$

Maggioriamo ora la somma dentro il segno di integrale. Sia $R$ un intero scelto tra $1,2, \ldots, n$; facendo variare $\theta$ in $\left(\theta_{R}-\frac{\theta_{1}}{2}, \theta_{R}+\frac{\theta_{1}}{2}\right),(R=1,2, \ldots n)$, si ha:

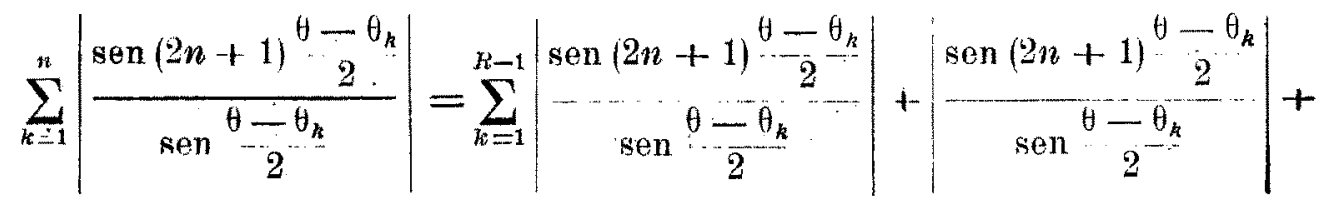

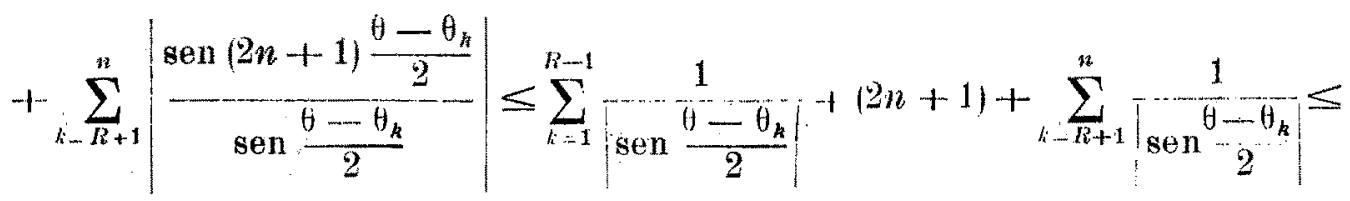

$$
\begin{aligned}
& \leq \sum_{k=1}^{n-1}\left|\operatorname{sen}\left(\theta_{R}-\frac{\theta_{1}}{2}-\theta_{k}\right) / 2\right|+(2 n+1) \sum_{k=R+1}^{n}\left|\operatorname{sen}\left(\theta_{R}+\frac{1}{\theta_{1}}-\theta_{k}\right) / 2\right| \leq \\
& \leq \pi \sum_{k=1}^{R-1}\left|\theta_{R}-\frac{1}{2}-\theta_{k}\right|+(2 n+1)+\pi \sum_{k=\left\{R_{1}+1\right.}^{n}\left|\begin{array}{c}
1 \\
\theta_{R}+\frac{\theta_{i}}{2}-\theta_{k}
\end{array}\right|= \\
& =\pi(2 n+1) \sum_{k=1}^{R-1} 2 R-2 k-\left.1\right|^{1}+(2 n+1)+\pi(2 n+1) \sum_{k=R+1}^{n}|2 k-2 k+1|^{\circ}
\end{aligned}
$$


Ed essendo $\sum_{s=1}^{R} \underset{s}{1}<C^{\prime} \log R$, con $C^{\prime}$ costante assoluta, sarà :

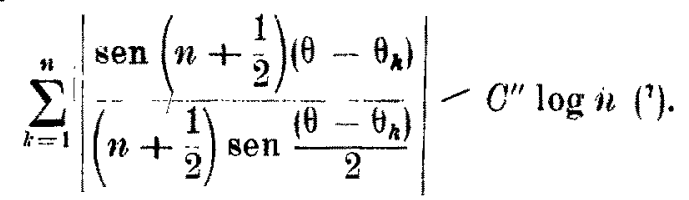

Ma è

$$
\int_{\frac{n_{1}}{2}}^{\pi} \frac{1}{\operatorname{sen}^{2} \theta} d \theta=2\left[-\operatorname{cotg} \frac{\theta}{4}\right]_{\frac{\theta_{1}}{2}}^{\pi}=2 \operatorname{cotg} \frac{\pi}{2(2 n+1)}<C^{\prime \prime \prime} \cdot n
$$

per cui sarà, tenuto conto della $(6)$ :

$$
I_{2}(n)<C_{2} n(\log n)^{4} . \quad \text { con } C_{2} \text { costante assoluta. }
$$

4. Tenuto conto delle (5) e (7), avremo:

$$
I(n) \leq C_{3} n(\log n)^{4} \text { con } C_{3} \text { costante assoluta ir. }
$$

Sarà allora, per la $\left(3^{\prime}\right)$, dipendente da $n$.

$$
\int_{-1}^{1} \sqrt{\frac{1-x}{1+x}}\left[f(x)-L_{n}(x)\right]^{4} d x \leq 8 \Delta^{4}\left[\pi+C_{3} n(\log n)^{4}\right] .
$$

Sia ora $f(x)$ lipschitziana di ordine $\alpha=\frac{1}{4}+\varepsilon, \varepsilon>0$, sarñ :

e quindi,

$$
\Delta=\mathrm{Cn}^{-\left(\frac{1}{4}+8\right)}\left({ }^{8}\right)
$$

$$
\int_{-1}^{1} \sqrt{\frac{1-x}{1+x}\left[f(x)-\left.L_{n}(x)\right|^{4} d x \leq \frac{8\left[\pi+C_{3} n(\log n)^{4}\right]}{C n^{1+4 \varepsilon}}\right.}, \quad \text { e. v. d. }
$$

(7) Cfr. D. JACKson. The Theory of approximation, "A.m. Math. Coll. Pabl. *, XI (1830), pag. 120.

(') (fr. loc. oit. (1), pagg. 48-49. 